\title{
The biometric study in different stages of primary angle- closure glaucoma
}

${ }^{1}$ Department of Public Health and Institute of Public Health, National Yang-Ming University, Taipei, Taiwan

${ }^{2}$ Department of Ophthalmology, Madou Sin-Lau Hospital, Tainan County, Taiwan

\section{${ }^{3}$ Department of}

Ophthalmology, Kaohsiung Veterans General Hospital, Kaohsiung, Taiwan

${ }^{4}$ School of Medicine, National Yang-Ming University, Taipei, Taiwan

Correspondence: P Chou, Community Medicine Research Center and Institute of Public Health, National Yang-Ming University, No.155, Sec. 2, Linong Street, Taipei 11221, Taiwan

Tel: +8862 28267050 ;

Fax: + 886228201461

E-mail: pschou@ym.edu.tw

Received: 10 May 2012 Accepted in revised form: 12 May 2013

Published online: 21 June 2013

\begin{abstract}
Purpose This study compared the general and ocular biometric characteristics of normal, primary angle closure (PAC), and primary angle-closure glaucoma (PACG) patients to better understand the possible relationship between differences in ocular parameters that might predict risk for PACG in PAC patients.

Methods One hundred normal, 90 PAC, and
\end{abstract} 90 PACG eyes were retrospectively reviewed. General characteristics such as age, gender, body height, body weight, blood pressure, pulse, systemic diseases, and education level were recorded. Ocular findings included visual acuity, intraocular pressure, refraction, cup to disc ratio, and ocular biometry. Ocular biometry was obtained by A-scan

ultrasonography (Digital A/B scan 5500; Sonomed Inc.). The parameters recorded were anterior chamber depth (ACD), lens thickness (LT), axial length (AXL), lens/axial length factor (LAF), and relative lens position (RLP).

Results Although the controls, PAC group, and PACG group were found to be significantly different in age $(62.7 \pm 9.8$; $65.3 \pm 7.5$; and $66.0 \pm 7.4$, respectively), there were no gender differences. With regard to ocular parameters, the ACD tended to decrease and the LT and LAF tended to increase from normal to PAC to PACG. The eyes of the PACG group had significantly shallower ACD $(P<0.001)$ and thicker lens $(P<0.001)$ than those of the PAC group. While PAC had similar lens position to the control group, PACG had more anteriorly positioned lens than the PAC group $(P<0.001)$. Logistic regression analysis found a significant association between a decrease in ACD and increased risk of PACG (odds ratio $(\mathrm{OR})=3.59$ for $0.2 \mathrm{~mm}$ decrease in $\mathrm{ACD}$ )
Y-Y Chen ${ }^{1,2}, Y-Y$ Chen $^{3,4}$, S-J Sheu ${ }^{3,4}$ and $\mathrm{PChou}{ }^{1}$ as well as a significant association between an increase in LT and increased risk of PACG $(\mathrm{OR}=1.30)$.

Conclusions In addition to LT, a shallower ACD owing to a change in RLP may have a role in the progression from PAC to PACG. Owing to the differences of certain biometric characteristics between PAC and PACG, A-scan ultrasonography might potentially be used for the early detection of PACG in PAC eyes.

Eye (2013) 27, 1070-1076; doi:10.1038/eye.2013.127; published online 21 June 2013

Keywords: A-scan ultrasonography; ocular biometry; primary angle-closure glaucoma; primary angle closure

Introduction

Primary angle-closure glaucoma (PACG), which is more common in Asia than in Western countries, causes severe deficits in visual function if it is not detected and treated early. ${ }^{1,2}$ People with PACG are at 2.5 times higher risk of blindness than those with primary open-angle glaucoma. ${ }^{1-3}$ Therefore, early detection of PACG is important. While gonioscopy examination is recognized as the gold standard for identifying individuals at risk for PACG, it requires highly trained ophthalmologists, who are in short supply in developing countries. However, A-scan ultrasonography, which is portable and noninvasive, can measure the ocular dimensions easily. ${ }^{4,5}$

Several studies have used A-scan ultrasonography to characterize PACG, most comparing the ocular dimensions between PACG and control eyes, and some comparing ocular biometry among subgroups of PACG. However, in these studies, the term 'glaucoma' 
was defined only by elevated IOP, meaning that an eye without visual field defect or optic neuropathy could be classified into glaucoma based on an IOP of over $20 \mathrm{~mm} \mathrm{Hg}$ alone. Some studies also divided PACG into subacute, acute, and chronic based on their clinical symptoms only. ${ }^{6-8}$ In this classification, an eye was defined as acute PACG because it had a sudden-onset IOP elevation, even if the eye could possibly resolve to normal function without any visual field or disc change. Therefore, the nomenclature could not represent the long-term severity of disease. Another problem with these studies is that they used different PACG classification systems, resulting in overlapping or confusing diagnostic criteria. Together, these problems explain the possible reason that the results of previous studies on the A-scan biometry of PACG were inconsistent and could not be compared.

In 2002, Foster et $a l^{9}$ proposed a new and stricter definition of PACG, which placed emphasis on structural and functional change, regardless of intraocular pressure. According to that classification system, if a patient has an eye with an occludable angle and a visual field defect compatible with glaucomatous optic neuropathy, he or she is diagnosed as having PACG. However, eyes with occludable angle and evidence of trabecular meshwork obstruction by the peripheral iris, but no damage to the visual field and optic nerve, are classified as having primary angle closure (PAC). Based on this definition, PAC is less severe than PACG and could be a possible precursor to PACG. While some studies have used A-scan ultrasonography to study eye structure of PACG, most have not adopted Foster's diagnostic criteria in their classifications. Therefore, in this study, we recorded general patient characteristics and used A-scan ultrasonography to characterize ocular biometry in normal, PAC, and PACG eye groups to identify what factors might increase the risk of PACG in PAC patients. The results of such a study might make possible the early detection of PACG and help delay the possible progression of the disease.

\section{Materials and methods}

Reviewing all the medical charts of a glaucoma specialist in our hospital between July 2009 and January 2010, we identified the patients with PAC or PACG as possible study subjects and identified patients with normal healthy eyes or those with only cataract as possible controls. We included all of those between 40 and 80 years old with a refractive error within $\pm 8 \mathrm{D}$ of the spherical equivalent and medical charts with complete ocular examination data. Both possible study subjects and possible controls were excluded if they had plateau iris or a history of acute angle-closure glaucoma (AACG), or had received peripheral laser iridotomies or intraocular surgery. Eyes with secondary glaucoma mechanisms were excluded. We also excluded those who were taking miotics because these drugs might confound the biometric data by moving the lens-iris diaphragm forward. Using a random table with odd numbers representing right eyes and even numbers left eyes, we chose one eye from each participant. In total, we enrolled 280 participants (90 with PACG, 90 with PAC, and 100 normal controls).

From the medical records, we collected general data such as age, sex, body height, body weight, and education level. We also recorded blood pressure data and whether the subject had a systemic disease (eg, diabetes or hypertension).

All patients had received visual acuity and refraction examinations. Silt lamp, direct ophthalmoscope, and gonioscopy examinations were performed by the same glaucoma specialist. Intraocular pressure was measured before pupil dilation by Goldmen applanation tonometry.

Patients were diagnosed as PAC and PACG groups based on the criteria proposed by Foster. ${ }^{9}$ An occludable angle was defined as one in which $>270^{\circ}$ circumference of the pigmented trabecular meshwork was invisible on gonioscopy. A patient was assigned to the PAC group if he or she had an occludable angle with features indicating trabecular obstruction, such as peripheral anterior synechiae (PAS), elevated intraocular pressure, or patchy pigmented deposition on the trabecular surface. Optic nerves and visual field had to be found to be normal by direct ophthalmoscopy and by visual field examination (Octopus perimeter, Interzeag Inc., Schlieren, Switzerland). A patient was assigned to the PACG group if his or her eye had an occludable angle and a visual field defect compatible with glaucomatous optic neuropathy. Optic neuropathy was defined as a vertical cup to disc ratio (CDR) $\geq 0.7$ or asymmetry $\geq 0.2$, or neuroretinal rim width reduced to $\leq 0.1 \mathrm{CDR}$. Two consecutive reliable visual field examinations performed by Octopus G1 program were required to prove significant glaucomatous defect, which was defined as a cluster of three contiguous points with $\geq 5 \mathrm{~dB}$ loss, two adjacent points with $\geq 10 \mathrm{~dB}$ loss or a difference of $\geq 10 \mathrm{~dB}$ across the nasal horizontal meridian at two adjacent points.

Ocular biometric data were collected by A-scan ultrasonography (Digital A/B scan 5500; Sonomed Inc., Lake Success, NY, USA) following standard procedures. Briefly, after topical anesthesia was applied, the ultrasound hand-held probe perpendicularly touched the center of the cornea. At least five measurements of anterior chamber depth (ACD), lens thickness (LT), and axial length (AXL) were taken. Excluding outliers, we averaged and recorded the most consistent readings. We 
further calculated the lens/axial length factor (LAF) using the formula LT $/ A X L \times 10$. As such, LAF could be regarded as a measure of relative LT. In addition, we calculated relative lens position (RLP) using Lowe's formula $(\mathrm{ACD}+0.5 \mathrm{LT}) / \mathrm{AXL} \times 10 .^{10}$

The patient characteristics and biometric data of all three groups were first summarized descriptively as mean and SD. One-way analysis of variance (ANOVA) was used to assess the overall differences among the three groups and Student's $t$-test to compare the PAC group with the other two groups. A $P$-value of $<0.05$ was considered significant. Furthermore, using logistic regression analysis, we attempted to identify the biometric parameters that might predict the risk of PACG in PAC patients. Finally, we plotted receiver operating characteristic curve (ROC curve) and calculated area under curve, sensitivity, and specificity. All statistical operations were performed using SPSS version 17.0 (SPSS Inc., Chicago, IL, USA).

\section{Results}

Of the 280 participants, 100 were the control, 90 were the PAC, and 90 were the PACG. The general and ocular characteristics of the eyes we studied are presented in Table 1. The overall differences among the three groups were assessed using one-way ANOVA and $P$-values were also reported in Table 1. Age was significantly different among the three groups (controls $62.7 \pm 9.8$ years; PAC 65.3 \pm 7.5 years; and PACG $66.0 \pm 7.4$ years) $(P<0.05)$, while there was no significant group differences in gender $(P=0.09)$. There were no significant differences in percentage of diabetes or hypertension, systolic pressure, diastolic pressure, or pulse rate. In all three groups, IOP was well controlled with a mean of 14-15 mm Hg. Although PAC and PACG patients were significantly shorter and lighter than the control group, there was no significant difference in body mass index $(P=0.12)$ among the three groups. As far as A-scan biometry and refraction data were concerned, the three groups had significantly different values. We could find the trend of descending ACD, increasing LT, and increasing LAF from control, PAC, to PACG. With regard to refraction, we found a trend PAC group to more hypermetropic than the controls but the PACG group to be less hypermetropic than the PAC group.

Table 2 compares these parameters in PAC with those of the other two groups with Student's t-test. We also

Table 1 General and ocular parameters for normal, priamry angle closure, and primary angle-closure glaucoma

\begin{tabular}{|c|c|c|c|c|}
\hline & $\begin{array}{c}\text { Control } \\
(\mathrm{n}=100)\end{array}$ & $\begin{array}{c}P A C \\
(\mathrm{n}=90)\end{array}$ & $\begin{array}{c}P A C G \\
(\mathrm{n}=90)\end{array}$ & P-value \\
\hline \multicolumn{5}{|l|}{ General characteristics } \\
\hline Age & $62.7 \pm 9.8$ & $65.3 \pm 7.5$ & $66.0 \pm 7.4$ & $P<0.05$ \\
\hline Gender & & & & $P=0.09$ \\
\hline Male $(n, \%)$ & $39(39.0 \%)$ & $23(25.6 \%)$ & $35(38.9 \%)$ & \\
\hline Female $(n, \%)$ & $61(61.0 \%)$ & $67(74.4 \%)$ & $55(61.1 \%)$ & \\
\hline Education (years) & $9.5 \pm 5.2$ & $7.7 \pm 4.7$ & $7.6 \pm 4.4$ & $P<0.05$ \\
\hline Body height $(\mathrm{cm})$ & $160.3 \pm 7.7$ & $156.6 \pm 8.0$ & $158.8 \pm 9.1$ & $P<0.05$ \\
\hline Body weight $(\mathrm{kg})$ & $63.4 \pm 10.4$ & $59.8 \pm 10.4$ & $59.6 \pm 10.7$ & $P<0.001$ \\
\hline Body mass index & $24.6 \pm 3.5$ & $24.3 \pm 3.8$ & $23.5 \pm 3.4$ & $P=0.12$ \\
\hline Diabetes mellitus (n, \%) & & & & $P=0.34$ \\
\hline No & $79(79.0 \%)$ & $78(86.7 \%)$ & $72(80.0 \%)$ & \\
\hline Yes & $21(21.0 \%)$ & $12(13.3 \%)$ & $18(20.0 \%)$ & \\
\hline Hypertension (n, \%) & & & & $P=0.73$ \\
\hline No & $65(65.0 \%)$ & $60(66.7 \%)$ & $55(61.1 \%)$ & \\
\hline Yes & $35(35.0 \%)$ & $30(33.3 \%)$ & $35(38.9 \%)$ & \\
\hline Systolic blood pressure $(\mathrm{mm} \mathrm{Hg})$ & $132.6 \pm 19.7$ & $130.9 \pm 19.2$ & $134.6 \pm 18.9$ & $P=0.44$ \\
\hline Diastolic blood pressure $(\mathrm{mm} \mathrm{Hg})$ & $81.3 \pm 13.7$ & $78.9 \pm 14.5$ & $79.6 \pm 10.6$ & $P=0.42$ \\
\hline Pulse & $77.3 \pm 11.9$ & $77.4 \pm 13.3$ & $77.8 \pm 13.0$ & $P=0.97$ \\
\hline \multicolumn{5}{|l|}{ Ocular characteristics } \\
\hline Anterior chamber depth (mm) & $3.11 \pm 0.29$ & $2.58 \pm 0.22$ & $2.32 \pm 0.17$ & $P<0.001$ \\
\hline Lens thickness (mm) & $4.53 \pm 0.43$ & $4.99 \pm 0.28$ & $5.13 \pm 0.36$ & $P<0.001$ \\
\hline Axial length (mm) & $23.76 \pm 1.01$ & $22.66 \pm 0.76$ & $22.68 \pm 0.80$ & $P<0.001$ \\
\hline Relative lens position & $2.26 \pm 0.13$ & $2.24 \pm 0.10$ & $2.15 \pm 0.08$ & $P<0.001$ \\
\hline Lens/axial length factor & $1.91 \pm 0.20$ & $2.20 \pm 0.14$ & $2.26 \pm 0.16$ & $P<0.001$ \\
\hline Cup to disc ratio & $0.37 \pm 0.08$ & $0.43 \pm 0.10$ & $0.65 \pm 0.15$ & $P<0.001$ \\
\hline Refractive error & $-0.23 \pm 1.95$ & $0.86 \pm 1.76$ & $0.29 \pm 2.15$ & $P<0.01$ \\
\hline Intraocular pressure $(\mathrm{mm} \mathrm{Hg})$ & $14.5 \pm 2.8$ & $14.5 \pm 2.7$ & $15.2 \pm 3.3$ & $P=0.2$ \\
\hline
\end{tabular}

Abbreviations: PAC, primary angle closure; PACG, priamry angle-closure glaucoma. 
Table 2 Comparision between control, PAC, and PACG eyes

\begin{tabular}{lccc}
\hline & $\begin{array}{c}P A C \text { vs } \\
\text { control }\end{array}$ & $\begin{array}{c}\text { PAC vs } \\
\text { PACG }\end{array}$ & $\begin{array}{c}P A C G \text { vs } \\
\text { control }\end{array}$ \\
\hline Age & $P<0.05$ & $\mathrm{NS}$ & $P<0.05$ \\
Gender & $\mathrm{NS}$ & $\mathrm{NS}$ & $\mathrm{NS}$ \\
Education (years) & $P<0.05$ & $\mathrm{NS}$ & $P<0.05$ \\
Body height (cm) & $P<0.01$ & $\mathrm{NS}$ & $P<0.05$ \\
Body weight (kg) & $P<0.05$ & $\mathrm{NS}$ & $P<0.05$ \\
Body mass index & $\mathrm{NS}$ & $\mathrm{NS}$ & $\mathrm{NS}$ \\
Anterior chamber depth (mm) & $P<0.001$ & $P<0.001$ & $P<0.001$ \\
Lens thickness (mm) & $P<0.001$ & $P<0.01$ & $P<0.001$ \\
Axial length (mm) & $P<0.001$ & $\mathrm{NS}$ & $P<0.001$ \\
Relative lens position & $\mathrm{NS}$ & $P<0.001$ & $P<0.001$ \\
Lens/axial length factor & $P<0.001$ & $P<0.05$ & $P<0.001$ \\
Cup to disc ratio & $P<0.001$ & $P<0.001$ & $P<0.001$ \\
Refractive error & $P<0.001$ & $\mathrm{NS}$ & $\mathrm{NS}$ \\
Intraocular pressure (mm $\mathrm{Hg})$ & $\mathrm{NS}$ & $\mathrm{NS}$ & $P<0.05$ \\
\hline
\end{tabular}

Abbreviations: NS, nonsignificant, defined as $P$-value $>0.05$; PAC, primary angle closure; PACG, priamry angle-closure glaucoma.

made the comparison between PACG and control group. PACG eyes were significantly different from the control group in every A-scan parameter but not in refraction. PAC eyes were significantly different from control eyes in ACD, LT, AXL, LAF, refraction $(P<0.001)$, but not in RLP. When PAC and PACG eyes were compared, AXL of the PAC eyes was similar to that of the PACG eyes. However, there was greater LT in the PACG group than in the PAC group $(P<0.01)$. Also, the relative $\mathrm{LT}$, expressed as $\mathrm{LAF}$, was significantly higher in PACG eyes than in the PAC group $(P<0.05)$. Furthermore, the PACG group had significantly shallower anterior chamber than the PAC group $(P<0.001)$. As for RLP, there was significant difference in this parameter between the PAC and PACG groups $(P<0.001)$, while the PAC and control groups had similar RLP.

Table 3, which summarizes age- and sex-adjusted odds ratio (OR) of the possible predictors of PACG in PAC patients, shows a significant association between decreased ACD and increased LT and a greater odds of developing PACG in PAC patients $(\mathrm{OR}=3.59$ for a $0.2 \mathrm{~mm}$ ACD decrease and $\mathrm{OR}=1.3$ for a $0.2 \mathrm{~mm} \mathrm{LT}$ increase). The only A-scan parameter not to be a significant risk predictor was AXL $(\mathrm{OR}=1.01 ; 95 \%$ confidence interval, 0.95-1.16).

Figure 1 shows the ROC curves for ACD, LT, AXL, RLP, and LAF in the detection of PACG in both PAC and PACG patients. We calculated AUC and summarized the findings in Table 4. The greatest AUC was achieved with ACD (AUC $=0.813)$. The next best performance was achieved by RLP (AUC = 0.736), followed by LT $(\mathrm{AUC}=0.661)$ and LAF $(\mathrm{AUC}=0.624)$. However, AXL performed poorly (AUC $=0.495)$. It can be learned that an $\mathrm{ACD}<2.46 \mathrm{~mm}$ would have a sensitivity of $65.6 \%$ and a specificity of $75.6 \%$.
Table 3 Computation of OR for PACG vs PAC, corresponding to a change of ocular biometry

\begin{tabular}{lcc}
\hline & OR & $95 \%$ CI \\
\hline $0.2 \mathrm{~mm}$ decrease in ACD & 3.59 & $2.41-5.37$ \\
$0.2 \mathrm{~mm}$ increase in LT & 1.30 & $1.05-1.60$ \\
$0.2 \mathrm{~mm}$ increase in AXL & 1.01 & $0.94-1.09$ \\
\hline
\end{tabular}

Abbreviations: $\mathrm{ACD}$, anterior chamber depth; $\mathrm{AXL}$, axial length; $\mathrm{CI}_{\text {, }}$ confidence interval; LAF, lens/axial length factor; LT, lens thickness; OR, odds ratio; RLP, ralative lens position.

Logistic regression adjusted for age and gender.

\section{Discussion}

This study showed that a decrease in ACD was associated with a greater odds of PACG $(\mathrm{OR}=3.59$ for a $0.2 \mathrm{~mm}$ decrease) and an increase in LT with a higher risk of PACG $(\mathrm{OR}=1.3$ for a $0.2 \mathrm{~mm}$ LT increase) (Table 3$)$.

The only A-scan parameter that was not a significant risk predictor was AXL $(\mathrm{OR}=1.01 ; 95 \%$ confidence interval, 0.95-1.16). The PACG group had a greater relative LT (LAF), a more anteriorly positioned lens, and a shallower anterior chamber than the PAC group.

PACG is a common form of glaucoma in Asians, Eskimos, and Inuits.,11-13 In previous studies comparing the ocular biometry of people with and without PACG eye ${ }^{10,14-19}$ those with PACG eye were found to have ocular features predisposing them to the disease. These features include a shallow anterior chamber, thick lens, anterior positioning of lens, shorter axial length, small corneal diameter, and a small corneal curvature radius. However, most of these biometric studies were conducted when glaucoma was defined only by elevated intraocular pressure regardless of structural or functional change. In 2002, Foster ${ }^{9}$ proposed a classification in which the term PACG was used only when there was disc and field damage. Adopting this classification, we investigated the differences in characteristics among a normal control group and PAC and PACG groups. Of specific concern was the ocular biometric differences between the PAC and PACG groups. To the best of our knowledge, this is the first study evaluating the differences of ocular parameters between PAC and PACG, based on the clear diagnostic distinction proposed by Foster.

In our study, PACG patients were significantly older than the controls, a finding consistent with other studies. PAC and PACG patients also had shorter body heights than the controls. Several studies have investigated the relationship between stature and ocular dimension. ${ }^{20-22}$ The Beijing Eye study reported a significant association between a shallow ACD and a narrow anterior chamber angle and short body stature. ${ }^{20}$ The Singapore Malay Eye study reported an association between longer AXL and greater body height. ${ }^{22}$ The Tanjong Pagar survey in Singapore also reported a significant association between 

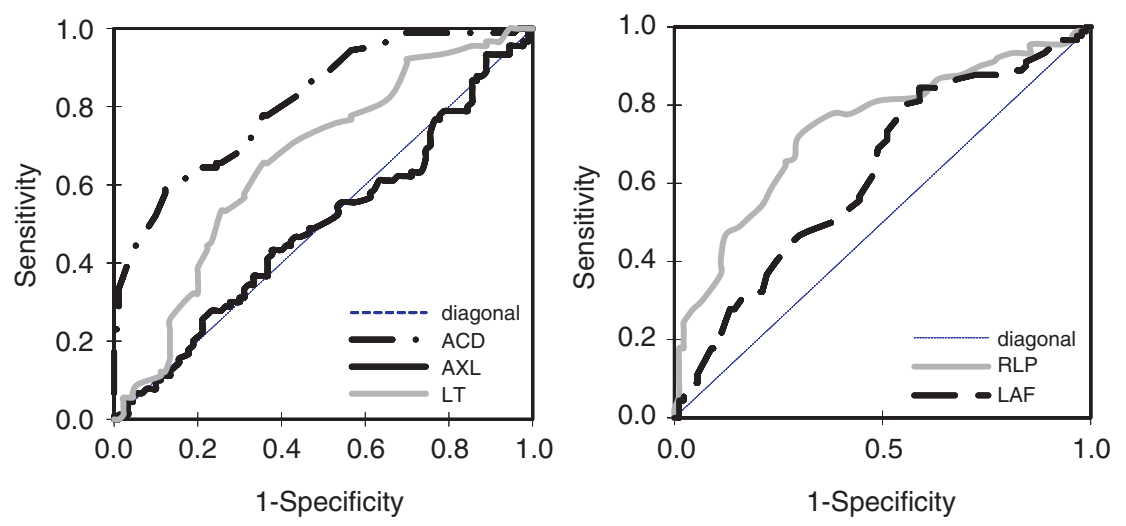

Figure 1 Receiver operating characteristic curve demonstrating performance of different parameters (ACD, LT, AXL, RLP, and LAF) in detecting PACG in both PAC and PACG patients.

Table 4 AUC for ACD, LT, AXL, RLP, and LAF in detecting PACG

\begin{tabular}{lcc}
\hline & AUC & 95\% CI of AUC \\
\hline ACD & 0.813 & $0.752-0.873$ \\
LT & 0.661 & $0.581-0.741$ \\
AXL & 0.495 & $0.410-0.580$ \\
RLP & 0.736 & $0.662-0.809$ \\
LAF & 0.624 & $0.542-0.705$ \\
\hline
\end{tabular}

Abbreviations: $\mathrm{ACD}$, anterior chamber depth; $\mathrm{AUC}$, area under curve $\mathrm{AXL}$, axial length; $\mathrm{CI}$, confidence interval; $\mathrm{LAF}$, lens/axial length factor; LT, lens thickness; RLP, ralative lens position.

adult shortness and shallower ACD. ${ }^{21}$ Thus, a shorter person may have smaller eyes, which might predispose them to angle closure. In our study, the mean ACD of PACG eyes was $2.32 \mathrm{~mm}$, which was $\sim 0.8 \mathrm{~mm}$ shallower than that of control eyes $(3.11 \mathrm{~mm})$. The mean LT of PACG was greater than $5.0 \mathrm{~mm}$ and that of the control eyes $4.5 \mathrm{~mm}$, while mean AXL in PACG $(22.68 \mathrm{~mm}$ ) was $1.1 \mathrm{~mm}$ less than that of the controls $(23.76 \mathrm{~mm})$. Previous studies in Taiwan conducted by Hung and Quigley had similar results. ${ }^{11,18}$ In addition, the biometric values of the PAC fell between those of the controls and the PACG group, suggesting that having PAC might increase the risk of developing PACG.

In order to identify which features predicted high-risk for PACG in those with PAC, we compared the ocular biometric measures of the PAC and PACG groups. While the two groups had similar AXL, the PACG group had significantly different RLP, shallower anterior chambers, thicker lens, and a greater LAF than the PAC group. The shallower ACD in PACG patients resulted from changes in LT and position, ${ }^{10,23}$ thus the two groups had significant differences in every lens-related parameter. Our computation of OR by logistic regression revealed ACD and LT to be significant predictors of PACG in people with PAC. The ROC curves confirmed that ACD was the best predictor.
We had another interesting finding about refraction of the control, PAC, and PACG groups. Previous studies have found that people with hypermetropia are at greater risk for angle closure. ${ }^{10,20,24}$ In our study, the mean refraction of the control, PAC, and PACG groups were $-0.23 \mathrm{D}$, $+0.86 \mathrm{D}$, and $+0.29 \mathrm{D}$, respectively, suggesting that our PAC was more hypermetropic than the control group $(P<0.001)$. We did not find trend toward hypermetropia between the PAC and PACG group. This lack of trend was because PACG patients had significantly thicker lens and more anterior lens position than the PAC group, but similar axial length. All these factors work together to induce a myopic shift, possibly masking the inherent trend toward hypermetropia.

These findings may be of clinical benefit. First, because our study revealed that PACG eyes tended to differ biometrically from PAC eyes, A-scan ulrasonography might potentially be used as a screening technique for prediction of disease progression. Second, in addition to LT, a shallower ACD owing to a change in RLP may have a role in the progression from PAC to PACG. Third, because lens position, as well as LT and relative LT (LAF), has an important role in differentiating PACG from PAC, a lensectomy might be considered in PAC patients if their biometric parameters predispose them to PACG, though the effect of lensectomy would need further study to verify its use.

This study has some limitations. The ocular hypertension in our study may be attributed to angle closure, thick cornea, or open-angle mechanism with potential PAC. The first entity is what we want to study, but the others may contaminate our results. In our study, we did not routinely check central corneal thickness (CCT). Therefore, some eyes, based only on the definition of occludable angle with IOP elevation, might have been classified as PAC when they might have been eyes of thick cornea. ${ }^{25-27}$ This misclassification might have biased our results. However, when reviewing our 
patients' data, we found that all the PAC and PACG patients had occludable angles with PAS or patchy pigmentation of trabecular meshwork. In other words, they showed anatomical evidence of angle closure, regardless of IOP. Thus, the confounding effect of thick cornea subjects should be minimal. In addition, when reviewing our patients' data, we found all PAC patients had normal IOP following further laser peripheral iridotomies. It implied that originally the elevation IOP was mostly owing to angle closure. Thus, the confounding effect of open angle with potential PAC should also be minimal.

Another limitation may be that we used A-scan ultrasonography to obtain ocular biometry. Therefore, our ACD value represented the distance from corneal epithelium to anterior lens capsule. Strictly speaking, the 'True' ACD, which is the distance from the corneal endothelium to anterior lens capsule, should be calculated by subtracting the CCT from the ACD. However, we did not routinely measure CCT with pachymetry, meaning that we could not obtain a true ACD (tACD) for our analysis. It was possible that our comparison results of ACD of the controls, PAC, and PACG groups may have been affected by variation in CCT. While this is possible, the Liwan eye study conducted in southern China showed CCT did not differ significantly in persons with PACG $(546 \pm 29 \mu \mathrm{m})$ and normal persons $(540 \pm 31 \mu \mathrm{m}){ }^{28}$ Pang et al ${ }^{29}$ and Aung colleagues have also suggested that the CCT values between PACG $(541.3 \pm 34 \mu \mathrm{m})$ and normal eyes $(536.7 \pm 31 \mu \mathrm{m})$ in Chinese subjects are not significantly different. Thus, we can have the idea that CCT is similar between angle-closure group and normal group. Therefore, in our study, the sequence of mean ACD for normal, PAC, and PACG group will not be changed after a similar value of CCT is subtracted from ACD. To confirm this was the case, we created a computergenerated random block of CCT. The CCTs for each group should approximate the normal distribution described in a previous study $(540 \pm 31 \mu \mathrm{m}$ in control group, $546 \pm 29 \mu \mathrm{m}$ in PAC group, and $546 \pm 29 \mu \mathrm{m}$ in PACG group). ${ }^{28}$ Thus, we could get the tACD of each subject and found the mean tACD would be $2.58 \pm 0.30$, $2.04 \pm 0.22$, and $1.78 \pm 0.18$ in normal, PAC, and PACG groups, respectively $(P<0.001)$. The PAC group had a significantly shallower tACD than controls $(P<0.001)$, as did the PACG group $(P<0.001)$. The PACG group also had a significantly shallower tACD than the PAC group $(P<0.001)$. Finally, according to logistic regression analysis, there was a significant association between decreased tACD and a greater risk of developing PACG in PAC patients $(\mathrm{OR}=3.60$ for a $0.2 \mathrm{~mm}$ tACD decrease, $P<0.001)$. Thus, what variation there might have been in CCT did not change our main findings in our study.
Nevertheless, future studies should measure CCT for sake of elegance and research conformity.

Still another limitation is our use of vertical CDR in the diagnostic criteria for glaucomatous optic neuropathy. Previous studies have reported interindividual variation in optic disc size and in number of axons in the optic nerve. ${ }^{30,31}$ Thus, the cutoff value of vertical CDR between normal and glaucoma is higher is large discs and lower in small discs. If a single cutoff value of vertical CDR is used, misclassifications will occur as a result of different disc sizes. Therefore, we also emphasized the configuration of neuroretinal rim notch, which means there was a focal reduction of neuroretinal rim width. The requirement of functional evidence of glaucoma in the form of a visual field defect would help reduce the possibility of misclassification.

As this study is a retrospective study, we cannot draw a definite causal relationship between biometric changes and progression from PAC to PACG. Further prospective studies are needed to confirm whether the differences we found in the biometric parameters can predict the likelihood that some people with PAC are at higher risk of developing PACG than others. And, further research is necessary to evaluate the effectiveness of A-scan biometry in screening for early PACG in PAC patients.

\section{Summary}

What was known before

- PACG eyes have shallower ACD, thick lens, anterior positioning of lens, and shorter acial length than the normal eyes.

What this study adds

- Adopting Fosters classification of PACG, we investigated the differences in A-scan characteristics among normal, PAC, and PACG groups.

- This is the first study evaluating the differences of ocular parameters between PAC and PACG, based on the Foster classification.

- We found the ocular biometric parameters might predict risk for PACG in PAC patients.

\section{Conflict of interest}

The authors declare no conflict of interest.

\section{References}

1 Foster PJ, Johnson GJ. Glaucoma in China: how big is the problem? Br J Ophthalmol 2001; 85(11): 1277-1282

2 Quigley HA, Broman AT. The number of people with glaucoma worldwide in 2010 and 2020. Br J Ophthalmol 2006; 90(3): 262-267. 
3 Foster PJ, Oen FT, Machin D, Ng TP, Devereux JG, Johnson GJ et al. The prevalence of glaucoma in Chinese residents of Singapore: a cross-sectional population survey of the Tanjong Pagar district. Arch Ophthalmol 2000; 118(8): 1105-1111.

4 Devereux JG, Foster PJ, Baasanhu J, Uranchimeg D, Lee PS, Erdenbeleig Tet al. Anterior chamber depth measurement as a screening tool for primary angle-closure glaucoma in an East Asian population. Arch Ophthalmol 2000; 118(2): 257-263.

5 Nolan WP, Aung T, Machin D, Khaw PT, Johnson GJ, Seah SK et al. Detection of narrow angles and established angle closure in Chinese residents of Singapore: potential screening tests. Am J Ophthalmol 2006; 141(5): 896-901.

6 Lan YW, Hsieh JW, Hung PT. Ocular biometry in acute and chronic angle-closure glaucoma. Ophthalmologica 2007; 221(6): 388-394.

7 Sihota R, Dada T, Gupta R, Lakshminarayan P, Pandey RM. Ultrasound biomicroscopy in the subtypes of primary angle closure glaucoma. J Glaucoma 2005; 14(5): 387-391.

8 Sihota R, Lakshmaiah NC, Agarwal HC, Pandey RM, Titiyal JS. Ocular parameters in the subgroups of angle closure glaucoma. Clin Experiment Ophthalmol 2000; 28(4): 253-258.

9 Foster PJ, Buhrmann R, Quigley HA, Johnson GJ. The definition and classification of glaucoma in prevalence surveys. Br J Ophthalmol 2002; 86(2): 238-242.

10 Lowe RF. Aetiology of the anatomical basis for primary angle-closure glaucoma. Biometrical comparisons between normal eyes and eyes with primary angle-closure glaucoma. Br J Ophthalmol 1970; 54(3): 161-169.

11 Congdon N, Wang F, Tielsch JM. Issues in the epidemiology and population-based screening of primary angle-closure glaucoma. Surv Ophthalmol 1992; 36(6): 411-423.

12 Sihota R, Agarwal HC. Profile of the subtypes of angle closure glaucoma in a tertiary hospital in north India. Indian J Ophthalmol 1998; 46(1): 25-29.

13 He M, Foster PJ, Ge J, Huang W, Zheng Y, Friedman DS et al. Prevalence and clinical characteristics of glaucoma in adult Chinese: a population-based study in Liwan District, Guangzhou. Invest Ophthalmol Vis Sci 2006; 47(7): 2782-2788.

14 Tomlinson A, Leighton DA. Ocular dimensions in the heredity of angle-closure glaucoma. Br J Ophthalmol 1973; 57(7): 475-486.

15 Lee DA, Brubaker RF, Ilstrup DM. Anterior chamber dimensions in patients with narrow angles and angleclosure glaucoma. Arch Ophthalmol 1984; 102(1): 46-50.

16 Salmon JF, Swanevelder SA, Donald MA. The dimensions of eyes with chronic angle-closure glaucoma. J Glaucoma 1994; 3(3): 237-243.

17 Hung PT, Hou YC, Lan WL. Chamber angle and biometric study in PACG and its lens. In: Krieglstein GK (ed). Glaucoma Update 5th Vol, Kaden Verlag: Germany, 1995, pp 309-314.
18 Hou YC, Hung PT, Lee YC. Biometric differences in normal, cataract and glaucoma subjects. J Med Ultrasound 1996; 4: 118-123.

19 Marchini G, Pagliarusco A, Toscano A, Tosi R, Brunelli C, Bonomi L. Ultrasound biomicroscopic and conventional ultrasonographic study of ocular dimensions in primary angle-closure glaucoma. Ophthalmology 1998; 105(11): 2091-2098.

$20 \mathrm{Xu}$ L, Cao WF, Wang YX, Chen CX, Jonas JB. Anterior chamber depth and chamber angle and their associations with ocular and general parameters: the Beijing Eye Study. Am J Ophthalmol 2008; 145(5): 929-936.

21 Chang L, Aung T, Low S, Wong TY, Khaw PT, Foster PJ. Is measurement of adult height useful in screening for primary angle closure? Eye (Lond) 2009; 23(9): 1775-1780

22 Lim LS, Saw SM, Jeganathan VS, Tay WT, Aung T, Tong L et al. Distribution and determinants of ocular biometric parameters in an Asian population: the Singapore Malay eye study. Invest Ophthalmol Vis Sci 2010; 51(1): 103-109.

23 Friedman DS, Gazzard G, Foster P, Devereux J, Broman A, Quigley $\mathrm{H}$ et al. Ultrasonographic biomicroscopy, Scheimpflug photography, and novel provocative tests in contralateral eyes of Chinese patients initially seen with acute angle closure. Arch Ophthalmol 2003; 121(5): 633-642.

24 Wilensky JT, Kaufman PL, Frohlichstein D, Gieser DK, Kass MA, Ritch R et al. Follow-up of angle-closure glaucoma suspects. Am J Ophthalmol 1993; 115(3): 338-346.

25 Copt RP, Thomas R, Mermoud A. Corneal thickness in ocular hypertension, primary open-angle glaucoma, and normal tension glaucoma. Arch Ophthalmol 1999; 117(1): 14-16.

26 Brandt JD, Beiser JA, Kass MA, Gordon MO. Central corneal thickness in the Ocular Hypertension Treatment Study (OHTS). Ophthalmology 2001; 108(10): 1779-1788.

27 Herman DC, Hodge DO, Bourne WM. Increased corneal thickness in patients with ocular hypertension. Arch Ophthalmol 2001; 119(3): 334-336.

28 Wang D, Huang W, Li Y, Zheng Y, Foster PJ, Congdon N et al. Intraocular pressure, central corneal thickness, and glaucoma in chinese adults: the liwan eye study. Am J Ophthalmol 2011; 152(3): 454-462.

29 Pang CE, Lee KY, Su DH, Htoon HM, Ng JY, Kumar RS et al. Central corneal thickness in Chinese subjects with primary angle closure glaucoma. J Glaucoma 2011; 20(7): 401-404.

30 Jonas JB, Gusek GC, Naumann GO. Optic disc, cup and neuroretinal rim size, configuration and correlations in normal eyes. Invest Ophthalmol Vis Sci 1988; 29(7): 1151-1158.

31 Jonas JB, Schmidt AM, Muller-Bergh JA, SchlotzerSchrehardt UM, Naumann GO. Human optic nerve fiber count and optic disc size. Invest Ophthalmol Vis Sci 1992; 33(6): 2012-2018. 